\title{
Article \\ Evaluation of Anesthetic Specific EEG Dynamics during State Transitions between Loss and Return of Responsiveness
}

\author{
Matthias Kreuzer ${ }^{\dagger}$, Tobias Kiel $^{\dagger}$, Leonie Ernst, Marlene Lipp, Gerhard Schneider (D) and Stefanie Pilge * \\ Department of Anesthesiology and Intensive Care, School of Medicine, Klinikum rechts der Isar, \\ Technical University of Munich, 81675 Munich, Germany; m.kreuzer@tum.de (M.K.); Tobias_Kiel@gmx.de (T.K.); \\ Leonie.Ernst@mri.tum.de (L.E.); marlene.lipp@tum.de (M.L.); gerhard.schneider@tum.de (G.S.) \\ * Correspondence: stefanie.pilge@tum.de \\ † These authors contributed equally to this work.
}

check for updates

Citation: Kreuzer, M.; Kiel, T.; Ernst, L.; Lipp, M.; Schneider, G.; Pilge, S. Evaluation of Anesthetic Specific EEG Dynamics during State Transitions between Loss and Return of Responsiveness. Brain Sci. 2022, 12, 37. https://doi.org/10.3390/ brainsci12010037

Academic Editor:

Giovanni Pellegrino

Received: 25 November 2021

Accepted: 23 December 2021

Published: 28 December 2021

Publisher's Note: MDPI stays neutral with regard to jurisdictional claims in published maps and institutional affiliations.

Copyright: (c) 2021 by the authors. Licensee MDPI, Basel, Switzerland. This article is an open access article distributed under the terms and conditions of the Creative Commons Attribution (CC BY) license (https:// creativecommons.org/licenses/by/ $4.0 /)$.

\begin{abstract}
Purpose: electroencephalographic (EEG) information is used to monitor the level of cortical depression of a patient undergoing surgical intervention under general anesthesia. The dynamic state transitions into and out of anesthetic-induced loss and return of responsiveness (LOR, ROR) present a possibility to evaluate the dynamics of the EEG induced by different substances. We evaluated changes in the EEG power spectrum during anesthesia emergence for three different anesthetic regimens. We also assessed the possible impact of these changes on processed EEG parameters such as the permutation entropy (PeEn) and the cerebral state index (CSI). Methods: we analyzed the EEG from 45 patients, equally assigned to three groups. All patients were induced with propofol and the groups differed by the maintenance anesthetic regimen, i.e., sevoflurane, isoflurane, or propofol. We evaluated the EEG and parameter dynamics during LOR and ROR. For the emergence period, we focused on possible differences in the EEG dynamics in the different groups. Results: depending on the substance, the EEG emergence patterns showed significant differences that led to a substance-specific early activation of higher frequencies as indicated by the "wake" CSI values that occurred minutes before ROR in the inhalational anesthetic groups. Conclusion: our results highlight substance-specific differences in the emergence from anesthesia that can influence the EEG-based monitoring that probably have to be considered in order to improve neuromonitoring during general anesthesia.
\end{abstract}

Keywords: anesthesia; anesthesia emergence; electroencephalogram; monitoring

\section{Introduction}

Patient monitoring based on the electroencephalogram (EEG) provides an easy-toapply approach in a clinical setting that may help to adjust anesthetic drugs to specific individual requirements for general anesthesia, i.e., to avoid too light or excessively deep anesthesia. Commercial devices, such as Bispectral Index (Medtronic, Dublin, Ireland) [1], SEDLine (Masimo, Irvine, CA, USA) [2], Entropy Module (GE Healthcare, Helsinki, Finland) [3], or Cerebral State Index (CSI) of the CSM monitor (Danmeter ApS, Odense, Denmark) [4] can provide a dimensionless index (e.g., from 0 to 100) that inversely correlates with the patients' level of cortical depression. It has to be mentioned that these indices rely on highly processed EEG information and hence it is not clear how they react to EEG changes right at a state transition or to substance specific differences. Inadequate anesthetic titration may severely influence patient outcome-too light anesthetic levels can lead to unintended wakefulness and too deep levels, especially with burst suppression EEG, may be associated with postoperative delirium (POD) [5,6]. Thus, the European Society of Anaesthesiology (ESA) evidence-based and consensus-based guidelines on POD recommend EEG based monitoring of anesthetic depth in all patients to avoid too deep general anesthesia, i.e., burst suppression [7]. While the raw EEG characteristics or the spectral 
representation of the EEG over time allows to understand the distinct, anesthetic-induced changes of this signal, the indices may not be able to fully reflect them. Further, known time delays in index calculation [8,9] can lead to unreliable indices during dynamic phases, such as anesthesia induction and emergence. Another issue are differences in EEG features that may also impact the one-fits-all indices. Different anesthetic substances induce different EEG patterns [10-12]. Demographic factors, such as age, alter the EEG characteristics and influence the indices [13-17]. When waking up from anesthesia the changes in EEG activity throughout emergence can follow different trajectories $[5,18,19]$. Because the type of trajectory seems to correlate with the risk of postoperative pain [18] or delirium in the postoperative care unit [5], monitoring the emergence phase is of special interest. Here, we present results from the analysis of a data set [20] that can help to understand the impact of anesthetic substance on processed EEG parameters, like spectral features, permutation entropy (PeEn) [21], and CSI [4] as a proxy for commercial monitoring applications.

\section{Materials and Methods}

\subsection{Study Design}

With our approach, we tried to analyze EEG behavior during the transitions between loss (LOR) and return of responsiveness (ROR). We chose to analyze the original data of a previous patient study, which is particularly suited to provide the key data (i.e., different anesthetics for maintenance of general anesthesia and accurate clinical verification of consciousness). We initially designed the study to evaluate the performance of the CSI during LOR and ROR. The Ethics Committee of the Technical University of Munich, Munich, Germany (Chairman Prof. A. Schömig) approved the study (Ethical Committee $\left.\mathrm{N}^{\circ} 1239 / 05\right)$ and we initiated the study on 2 March 2005. We included 45 adult patients with an American Society of Anesthesiologists physical status I or II, undergoing elective surgery under general anesthesia. All patients gave informed written consent to the study.

We defined the following exclusion criteria: history of neurological or psychiatric disease, medication known to affect the central nervous system including drug or alcohol abuse, and the indication of a rapid sequence induction (e.g., pregnancy, emergency). According to standard clinical practice, each patient received one of three pre-defined combinations of anesthetic drugs, our three study groups: sevoflurane group (sevoflurane/sufentanil), isoflurane group (isoflurane/sufentanil), and propofol group (propofol/remifentanil) with $n=15$ patients each. The attending anesthetist chose the anesthetic, relying on standard operating procedures for the particular surgical types, and in consideration of preexisting conditions. We used propofol for induction in all patients. We administered atracurium or mivacurium as neuromuscular blocking agent. There was no randomization in order to reflect standard clinical practice.

\subsection{Monitoring}

We used a Datex ${ }^{\circledR}$ AS/3 (GE Healthcare, Chalfont St Giles, United Kingdom) compact monitor for non-invasive measurement of blood pressure, heart rate, oxygen saturation, inspiratory oxygen, end-tidal carbon dioxide, and volatile anesthetic concentrations and recorded demographic data and type of anesthetic premedication for each patient. For EEG recording and evaluation of index performance, we used a CSI monitor.

\subsection{Clinical Protocol}

Some patients received benzodiazepines for premedication according to clinical standard. The clinician in charge slowly induced anesthesia by intravenous injections of $20 \mathrm{mg}$ propofol every $30 \mathrm{~s}$. In order to define LOR and ROR, we asked the patient every $15 \mathrm{~s}$ to squeeze the investigator's hand during anesthesia induction and emergence. In case of a positive response, we verified the response by an immediate repetition of the command. We defined LOR as the first missing response after a repeated command during induction and ROR as the first positive response during emergence. In case the patient showed a reproducible response, i.e., opening of the eyes or moving of the head, but not reaction to 
the "squeeze hand" command, this time was noted ROR. To ensure a preserved ability to react to the command in case of administered neuromuscular blocking agents, we applied Tunstall's isolated forearm technique [22]. We delivered propofol, sevoflurane, or isoflurane for anesthesia maintenance.

Clinical endpoints were a constant end-tidal volatile anesthetic concentration and respectively a constant propofol effect-side concentration determined with the pharmacokinetic model by Schnider et al. [23].

\subsection{EEG Recording and CSI Calculation}

For our analyses, we stored the CSI trend data as well as the raw EEG as provided by the CSM monitor. The stored raw EEG had a sample rate of $100 \mathrm{~Hz}$ and a frequency range from 6-42 Hz. We chose electrode positions as recommended by the manufacturer: at the middle of the forehead, at the left forehead and at the mastoid region on the left side, all with impedances of $5 \mathrm{k} \Omega$ or less (https: / / www.danmeter.dk/en/files/CSM-MonitorMKII---Manual-561105003--US-only-.pdf accessed on 25 November 2021). We also stored the CSI, the burst suppression ratio (BS\%), and the signal quality data on a laptop computer equipped with specific software (CSM-Capture ${ }^{\circledR}$ 2.02, Danmeter, Odense, Denmark) using CSM Link ${ }^{\mathrm{TM}}$. The CSI is derived by a proprietary algorithm that utilizes an adaptive neurofuzzy inference system (ANFIS) to combine the information from the three spectral EEG parameters $\alpha$-ratio, $\beta$-ratio, and $\beta$-ratio/ $\alpha$-ratio as well as BS\% [4].

We recorded EEG and all related data synchronized to standard monitoring parameters and manually documented singular events like LOR/ROR in NeuMonD [24].

\subsection{CSI and EEG Analysis}

For our analyses that mainly focused on the EEG (and CSI) features during substancespecific anesthesia emergence, we calculated the power spectral density (PSD) for $10 \mathrm{~s}$ EEG episodes with a $1 \mathrm{~s}$ shift to construct density spectral arrays (DSA) and to derive the trends of power in the different EEG frequency bands. We further calculated the PeEn [21], a time domain measure that can be correlated to the power spectrum [25] of the recorded EEG. PeEn is one of the most powerful 1-channel EEG parameters to differentiate between consciousness and unconsciousness [26,27]. We calculated PeEn with embedding dimension $\mathrm{m}=3$ and time lag $\tau=1$ after a low pass filtering of the EEG to $30 \mathrm{~Hz}$ for $10 \mathrm{~s}$ EEG episodes with a $1 \mathrm{~s}$ shift as well. Because of the narrow EEG-bandwidth provided by the CSM, we confine our reporting to relevant findings in the 6-30 Hz range. We chose these settings for $\mathrm{m}$ and $\tau$ for PeEn calculation because of the direct link to the power spectrum [25].

We evaluated the performance of the parameters over the induction and throughout the emergence phase as well as the performance of PeEn and CSI to distinguish between a responsive and an unresponsive patient right at the state transitions LOR and ROR. Therefore, we compared the parameter values derived $15 \mathrm{~s}$ before the LOR/ROR event to the values $30 \mathrm{~s}$ after the event. The interval of $15 \mathrm{~s}$ to ask patients to squeeze hand allows a clear definition of LOR/ROR, and thus the conclusion that $15 \mathrm{~s}$ before LOR (ROR) the patient was responsive (unresponsive). We selected the index values $+30 \mathrm{~s}$ after the event to account for the time delay of index calculation [8], i.e., to allow the EEG monitor to indicate the current clinical state. This approach is consistent with previously published data [28,29]. It has to be mentioned that the derived results between CSI and PeEn are not comparable because of a varying and considerable time delay in index calculation of the CSI $[8,9]$.

\subsection{Statistical Analysis}

To evaluate spectral differences, we used the area under the receiver operating curve (AUC) together with 10k-fold bootstrapped 95\% confidence intervals. In case the 95\% confidence interval did not contain 0.5 (i.e., 'no effect'), the comparison was defined to be significantly different. Hentschke and Stuettgen also showed this relationship [30]. To avoid the discussion of false positives, we only considered findings significant if, in case of 
comparing PSD, at least two neighboring frequency bins showed significant differences. This or a similar approach has been used before $[10,14]$. For comparison of the density spectral arrays, we only discuss differences if they occurred in clusters, because of the low probability of a concentrated occurrence of spurious false positives in a cluster.

We used the Wilcoxon signed rank test (significance level: $p<0.05$ ) and the effect size Hedges' $\mathrm{g}$ for dependent data to test for differences in CSI and PeEn before and after LOR/ROR. We also used the AUC and 95\% confidence intervals to evaluate the parameters' (PeEn and CSI) performance to separate between responsiveness and unresponsiveness, because the AUC for dichotomous data is equivalent to the prediction probability Pk, commonly used to assess the performance of neuromonitoring approaches [31]. For analysis of the demographic data, the duration of CSI $\geq 80$ before ROR, and the differences in EEGband power during anesthesia emergence and ROR, we used a Kruskal-Wallis test with Dunn's post-hoc test to correct for multiple comparisons [32]. For comparison of sex and ASA distribution between groups, we used a Freeman-Halton test. We performed all statistical tests with MATLAB also using the MES toolbox [30].

\section{Results}

\subsection{Demographics}

There were no significant differences between the groups in age, size, gender, and ASA status. Patients in the isoflurane group had a significantly higher weight than patients in the sevoflurane $(p=0.036)$ and propofol $(p=0.002)$ group, but there was no significant difference in BMI among the groups. Table 1 contains the detailed information regarding the demographics. In the isoflurane group, 12 patients received dormicum, (+ additional tranxilium in six cases and one patient received extra nexium) and in the sevoflurane and propofol group, 11 patients each received dormicum (+ two received additional tranxilium and one extra nexium) for premedication. The duration from the begin of emergence period to ROR was $20 \mathrm{~min} 36 \mathrm{~s}$ (11 $\min 14 \mathrm{~s}-49 \mathrm{~min} 48 \mathrm{~s}$ ) for isoflurane, $16 \min 29 \mathrm{sec}(9 \mathrm{~min} 55 \mathrm{~s}$ $-39 \mathrm{~min} 05 \mathrm{~s}$ ) for sevoflurane and $14 \mathrm{~min} 28 \mathrm{sec}(7 \mathrm{~min} 0 \mathrm{~s}-27 \mathrm{~min} 30)$ for propofol.

Table 1. Patient characteristics data of the three patient groups.

\begin{tabular}{|c|c|c|c|c|}
\hline & $\begin{array}{c}\text { Propofol Group } \\
n=15\end{array}$ & $\begin{array}{l}\text { Sevoflurane Group } \\
\qquad n=15\end{array}$ & $\begin{array}{l}\text { Isoflurane Group } \\
\qquad n=15\end{array}$ & $p$-Value \\
\hline Age (y) & $57(4567)$ & $43(38.563 .5)$ & $40(3346.5)$ & $* p=0.1008$ \\
\hline Size $(\mathrm{cm})$ & $168(161$ 178.5) & 173 (167 179) & $180(173$ 185.5) & $* p=0.1059$ \\
\hline Weight (kg) & $71(6877)$ & 75 (69.5 79.5) & $90(81$ 100) & ${ }^{*} p=0.0021$ \\
\hline BMI & $25.4(23.2$ 31.2) & $25.8(23.836 .6)$ & $28.4(25.834 .1)$ & $* \overline{p=0.1474}$ \\
\hline LOC to ROC (min) & $107(89115)$ & $109(93182)$ & $129(103$ 142) & $* p=0.221$ \\
\hline Sufentanil ( $\mu \mathrm{g})$ & $\mathrm{n} / \mathrm{a}$ & $25.0(20.037 .5)$ & $30.0(27.540 .0)$ & $+p=0.391$ \\
\hline Remifentanil $(\mu \mathrm{g})$ & 992 (867 1408) & & & \\
\hline Gender $(\mathrm{m} / \mathrm{f})$ & $7 / 8$ & $9 / 6$ & $11 / 4$ & $\S p=0.3296$ \\
\hline ASA (I/II) & $10 / 5$ & $8 / 7$ & $10 / 5$ & $\S p=0.6839$ \\
\hline
\end{tabular}

Data are presented as mean (first and third quartiles). ${ }^{*}$ Kruskal-Wallis test; + Mann-Whitney U test; ${ }^{\S}$ FreemanHalton test.

\subsection{EEG and Index Features during Propofol-Induced Loss of Responsiveness}

The EEG during the propofol induced LOR showed the characteristic slowing of the EEG and the onset of strong oscillatory activity in the EEG alpha-band. Figure 1A shows the median DSA during LOR for all patients. When looking at certain time points before and after LOR, i.e., when comparing the power spectral density (PSD) extracted $15 \mathrm{~s}$ before LOR to the PSD $30 \mathrm{~s}$ after the event, we could observe significantly higher alpha power and lower power in the beta band frequencies after LOR (Figure 1B). These changes in the EEG impacted the processed EEG parameters PeEn and CSI, which significantly and strongly decreased during LOR (PeEn: $n=45 ; p<0.001, \mathrm{~g}=0.94$ (0.65 1.8); CSI: $n=43 ; p<0.001$, $\mathrm{g}=1.13$ (0.85 1.519) (Figure 1C). 


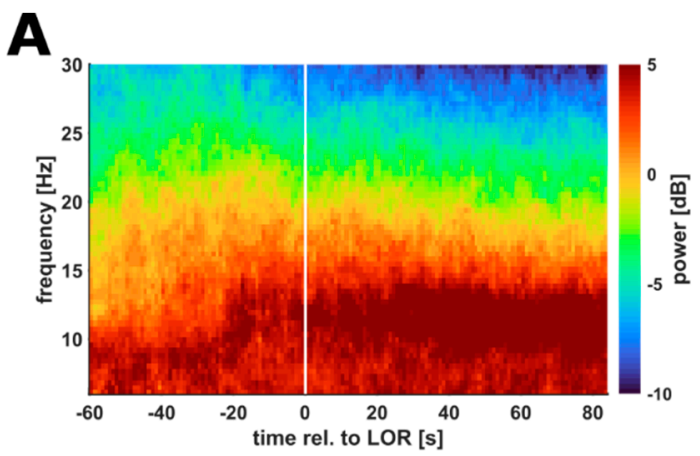

B
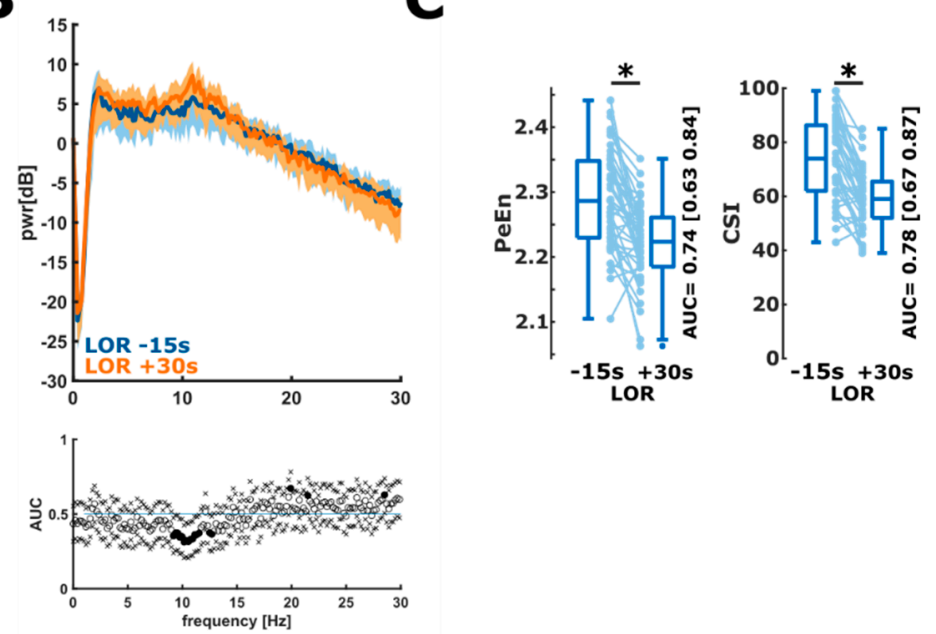

Figure 1. Changes in the spectral EEG representation and in processed EEG parameters during LOR. (A). Density spectral array (DSA) during the LOR episode. Over time, a strong alpha rhythm develops and the power in the faster frequencies decreases. (B) Comparison of the power spectral density $15 \mathrm{~s}$ before LOR (blue) to $30 \mathrm{~s}$ after LOR (orange). After LOR, the power in the alpha frequencies was significantly higher and there was power in the higher (beta) frequencies as indicated by the black dots in the AUC plot. (C) PeEn (left) significantly $\left(^{*}\right)$ and strongly decreased during LOR $(p<0.001$, $\mathrm{g}=0.94(0.651 .8)$ and the performance to separate responsiveness from unresponsiveness was "fair" $(\mathrm{AUC}=0.74(0.630 .84))$. CSI (right) significantly and strongly decreased during LOR $(p<0.001$, $\mathrm{g}=1.13(0.851 .51))$ and the performance of the CSI to separate responsiveness from un responsiveness was "fair" (AUC = $0.78(0.670 .87)$ ).

We also compared the spectral parameters between the patients in the different maintenance anesthetic regimen groups to ensure comparable conditions. We did not find significant differences between the groups as displayed in supplemental Figure S1. Table 2 contains the statistical details.

Table 2. Statistical parameters for the cerebral state index (CSI) and permutation entropy (PeEn) analysis at loss and return of responsiveness (LOR/ROR).

\begin{tabular}{cccccc}
\hline & $\mathbf{- 1 5} \mathbf{~ s}$ & $\mathbf{+ 3 0 ~ s}$ & $p$-Value & Hedge's g & AUC \\
\hline LOR: CSI & $74[62$ 86] & $59[52$ 65] & $\underline{p<0.001}$ & $1.13[0.851 .51]$ & $0.78[0.670 .87]$ \\
LOR: PeEn & $2.29[2.232 .35]$ & $2.22[2.192 .16]$ & $\underline{p<0.001}$ & $0.94[0.651 .8]$ & $0.74[0.630 .84]$ \\
ROR-isoflurane: CSI & $88(48$ to 93) & $87(81$ to 96$)$ & 0.234 & n.s. & $0.50[0.280 .74]$ \\
ROR-sevoflurane: CSI & $87(49$ to 97$)$ & $88(59$ to 96$)$ & 0.445 & n.s. & $0.59[0.360 .81]$ \\
ROR-propofol: CSI & $85.5(41$ to 99) & $87(78$ to 100$)$ & $\underline{0.023}$ & $-0.71[-1.20-0.33]$ & $0.63[0.170 .58]$ \\
ROR-isoflurane: PeEn & $2.36[2.332 .43]$ & $2.41[2.392 .44]$ & 0.057 & $0.52[-1.22-0.03]$ & $0.63[0.400 .85]$ \\
ROR-sevoflurane: PeEn & $2.43[2.252 .48]$ & $2.43[2.412 .46]$ & 0.216 & n.s. & $0.56[0.320 .78]$ \\
ROR-propofol: PeEn & $2.40[2.282 .44]$ & $2.44[2.392 .48]$ & 0.058 & $-0.68[-1.32-0.17]$ & $0.68[0.440 .85]$ \\
\hline
\end{tabular}




\subsection{Substance Specific Differences in the EEG and CSI Features during ROR}

We found differences in the EEG spectrum and the processed EEG parameters during anesthesia emergence towards ROR. Figure 2 displays the DSA from $10 \mathrm{~min}$ before until ROR for the three anesthetic groups as well as the statistical differences between the groups. At the start of emergence all groups show strong oscillatory activity in the EEG alpha-band, but in contrast to propofol, the patients receiving volatiles had higher power in the higher frequencies as well. This higher power persisted throughout emergence. While towards the end of emergence at around $2 \mathrm{~min}$ before ROR there were no more differences between the propofol and sevoflurane group, the differences between propofol and isoflurane remained (in parts until immediately before ROR). Between the isoflurane and sevoflurane group, we did not observe any significant differences between emergences (Figure 2). To complete the picture, we also assessed the differences in the normalized power and found the results to be weaker than for the absolute power. The corresponding plots can be found in supplemental Figure S2.

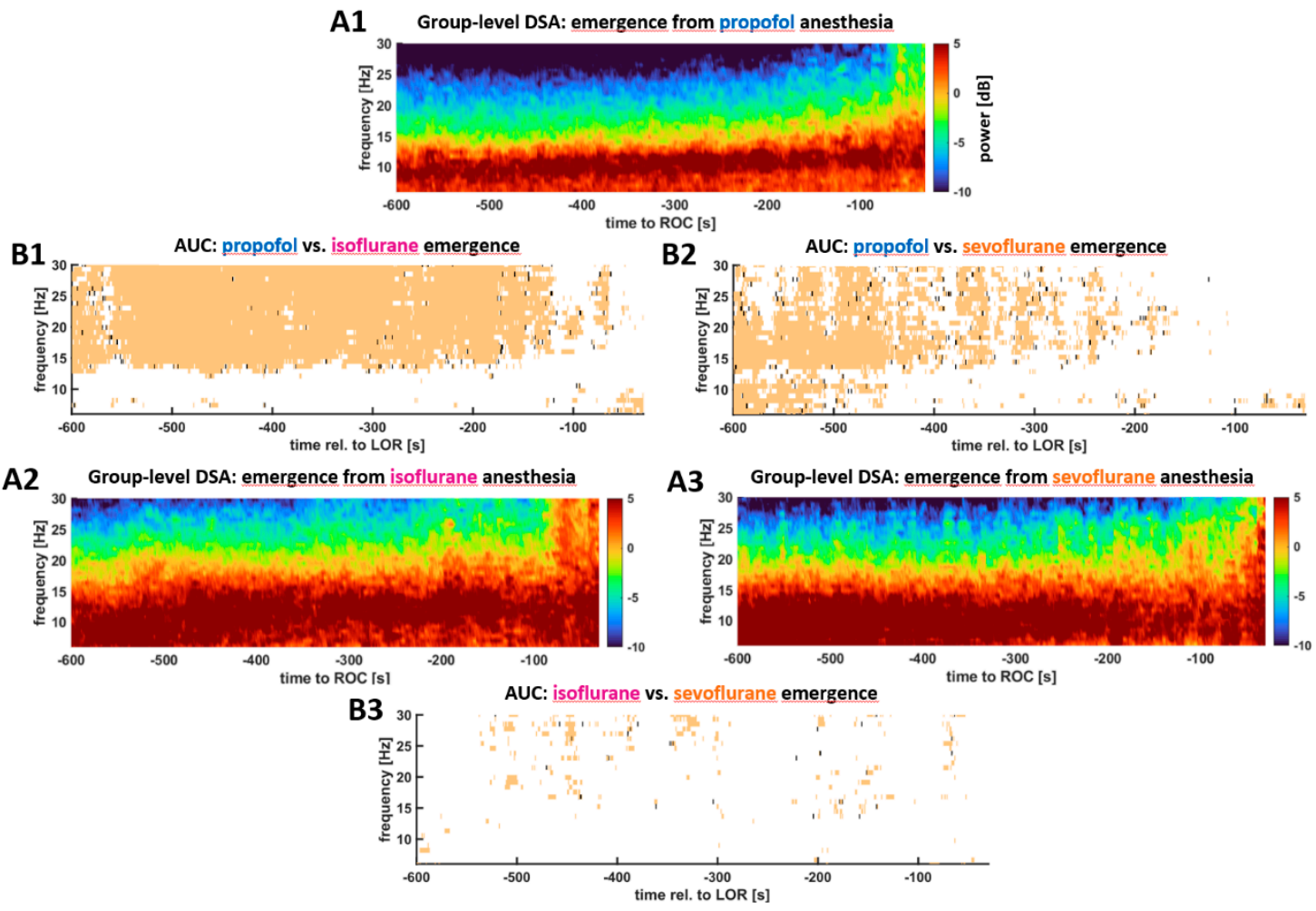

Figure 2. Density spectral arrays (DSA) plots for all three groups derived from the pooled (median) power spectral density of all patients within a group (A) and the corresponding information regarding a significant difference (B) as determined by the $95 \%$ confidence intervals of the AUC. A. DSA of anesthesia emergence for the different substance groups; A1. Group-level emergence DSA of the patients receiving propofol. A2. Group-level emergence DSA of the patients receiving isoflurane. A3. Group-level emergence DSA of the patients receiving sevoflurane. B. Maps of the pixel-wise AUC calculations. B1. Comparison between the propofol and isoflurane group. B2. Comparison between the propofol and sevoflurane group. B3. Comparison between the isoflurane and sevoflurane group. During emergence from propofol maintenance, the power in the frequencies above $\sim 15 \mathrm{~Hz}$ was significantly lower than from maintenance with inhalational anesthetics (B1,B2). These differences persisted until very close to ROR when compared to the isoflurane group (B2). There were no significant differences in the emergence behavior between sevoflurane and isoflurane (B3).

The difference in EEG changes towards ROR was also reflected in the behavior of the processed EEG parameters. For PeEn, we observed no significant change throughout the immediate transition to ROR. For isoflurane and propofol the PeEn did not increase 
significantly (isoflurane: $p=0.057$ propofol: $p=0.058$ ), but the effect size Hedges' $\mathrm{g}$ indicated a medium effect without the $95 \%$ CI containing 0 (isoflurane: $g=-0.52(-1.22-0.03$ ); propofol: $\mathrm{g}=-0.68(-1.32-0.17))$. There was no significant effect in the sevoflurane group $(p=0.216)$. The details are presented in Figure 3A-C. For the CSI, we did not find a significant change in the index from $15 \mathrm{~s}$ before until $30 \mathrm{~s}$ after ROR for the patients in the isoflurane $(p=0.234)$ and sevoflurane $(p=0.445)$ group. For propofol, we found a significant $(p=0.023)$ CSI increase of medium strength $(g=-0.71(-1.20-0.33))$. Figure 3D-F presents the corresponding connected dot and box plots. Table 2 presents the details. We also found significantly higher CSI and PeEn values $(p<0.001)$ after ROR than before LOR. The corresponding plots are presented in the supplements as Figure S4.
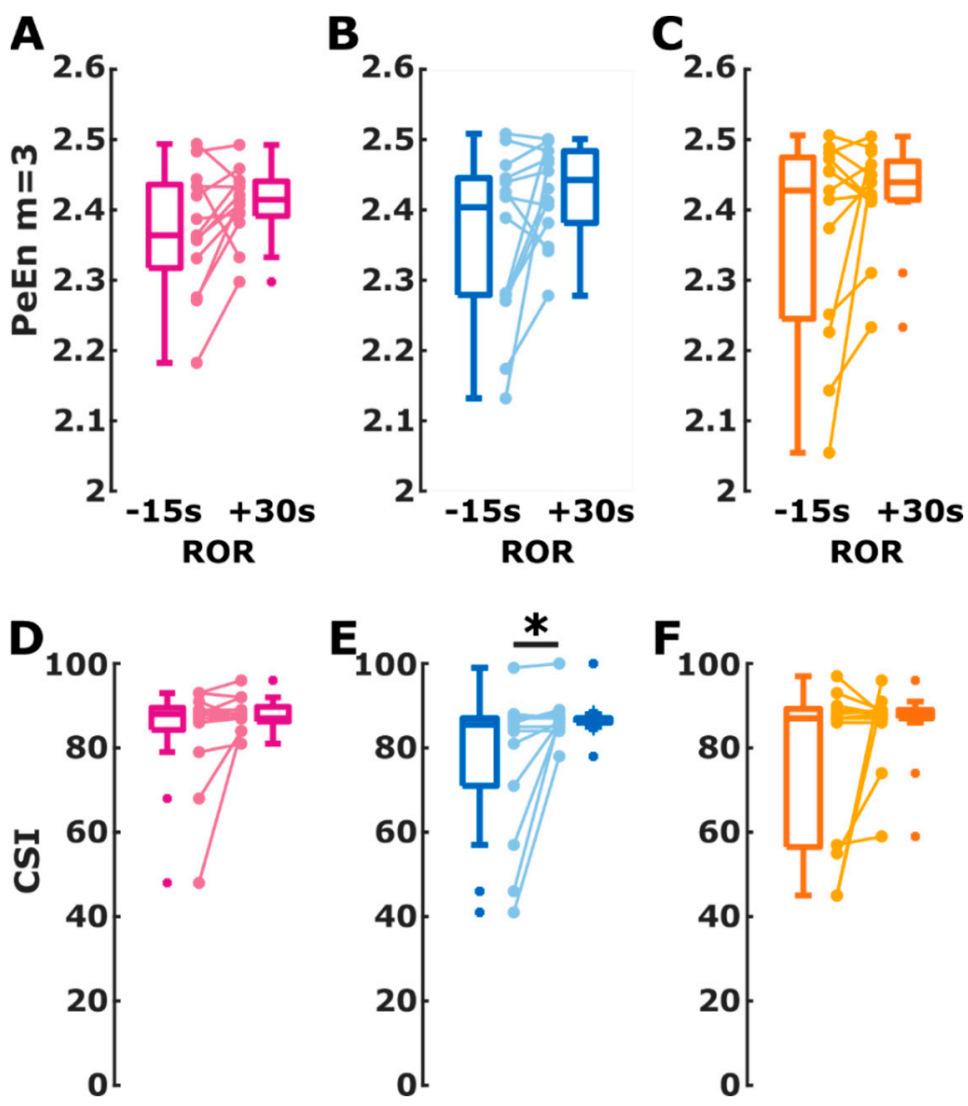

Figure 3. Change in cerebral state index (CSI) and permutation entropy (PeEn) values during return of responsiveness (ROR) from $15 \mathrm{~s}$ before ROR to $30 \mathrm{~s}$ after ROR for the isoflurane group (purple), propofol group (blue), and sevoflurane group (orange). The box plots indicate the distribution of CSI and PeEn values before and after ROR used for AUC calculation. The connected dots present the change in CSI and PeEn for each single patient. (A) The signed rank test indicated a non-significant change with a medium effect size of PeEn in patients assigned to the isoflurane group $(p=0.057$; Hedge's $g=-0.52(-1.22-0.03))$. (B) In the propofol group, the PeEn of the patients showed a non-significant $(p=0.0580)$ increase of medium effect $(g=-0.68(-1.32-0.17))$. (C) PeEn in the sevoflurane group increased non-significantly with a medium effect $(p=0.2163 ; \mathrm{g}=-0.77(-10.05)$ ). (D) There was no significant different in the CSI change within the patients for the isoflurane group $(p=0.2344)(E)$ The CSI in patients in the propofol group significantly $\left(^{*}\right)$ increased $(p=0.0234$; $\mathrm{g}=-0.71(-1.20-0.33)$, medium effect) (F) For the sevoflurane group the change within the patients was not significant $(p=0.4453)$.

\subsection{Implications of Substance-Specific Differences on Monitoring}

The earlier activation of higher EEG frequencies during anesthesia emergence influenced the CSI, used as a proxy for the monitoring systems. The median duration the CSI reflected wakefulness, CSI $\geq 80$, before ROR was 73 s [43 s 203 s] for isoflurane, 
$101.5 \mathrm{~s}$ [0 s $143.5 \mathrm{~s}$ ] for sevoflurane, and $24 \mathrm{~s}$ [ $2 \mathrm{~s} 51 \mathrm{~s}$ ] for propofol. There was no significant difference between the durations $(p=0.3145$, Chi-square $=2.31)$. Three patients in the isoflurane, four patients in the sevoflurane group, and two patients in the propofol group had CSI $<80$ at the time of ROR, included in the analysis as a duration of $0 \mathrm{~s}$. After removing these patients from this analysis, the median duration was $190.5 \mathrm{~s}(64.8 \mathrm{~s} 289.3 \mathrm{~s})$ for isoflurane, $143.5 \mathrm{~s}(101.5 \mathrm{~s} 222.5 \mathrm{~s})$ for sevoflurane, and $25 \mathrm{~s} \mathrm{(4} \mathrm{s} 79.5 \mathrm{~s})$ for propofol. These durations were significantly different $(p=0.0145$, Chi-square $=8.46)$ with a significant shorter duration of CSI $\geq 80$ for propofol when compared to isoflurane $(p<0.001$, AUC $=0.83(0.550 .98))$. There was no significant difference between the sevoflurane and propofol group, but the AUC $=0.80(0.5797)$ revealed a strong effect of substance on CSI $\geq 80$ before ROC. Figure 4 presents the according box plot.

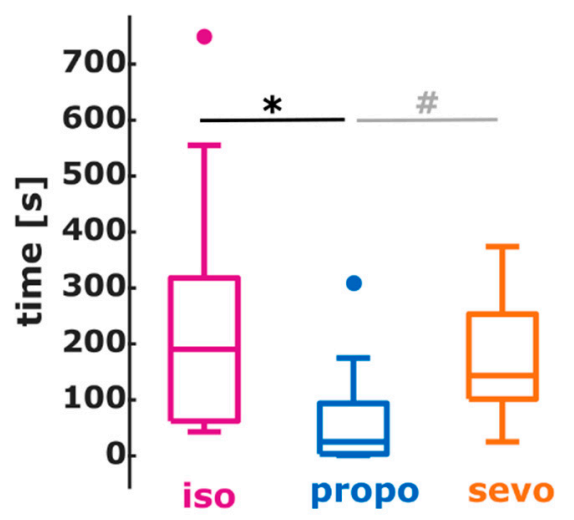

Figure 4. Boxplots representing the duration, the CSI indicated an awake patient before the return of responsiveness (ROR) for isoflurane (purple), sevoflurane (orange), and propofol (blue). For propofol, the duration was shortest with a significant $\left({ }^{*}, p<0.001\right)$ difference compared to isoflurane and a (\#, non-significant) but strong (AUC > 0.7) difference compared to sevoflurane.

\section{Discussion}

With our analyses, we could show that the EEG changes during anesthesia emergence and throughout the return to responsiveness depend on the maintenance anesthetic regimen. To investigate differences, we concentrated on the spectral changes in the EEG, from 10 min before ROR until ROR, as well as on the behavior of processed EEG indices, PeEn and CSI, immediately around ROR. In addition to the ROR transition for three anesthetic regimens, we also investigated the EEG changes during anesthesia induction with propofol. For the induction, we could observe slowing of EEG [33] and the development of strong oscillatory activity in the EEG alpha band, typical for propofol [10]. This change of the EEG towards slower oscillatory throughout LOR activity led to the significant decrease of PeEn and CSI. The LOR, in general, is a relatively fast process, triggered by administration of hypnotic agents (propofol) directly into the central compartment. Rapidly increasing effectsite concentrations affect critical structures in a way that leads to unconsciousness and that promptly modifies the EEG, facilitating relatively profound deviations in EEG-based parameters and indices as observed within the predefined time interval (e.g., LOR-15s to LOR+30s). We also used the LOR transition to check for the absence of significant differences in the EEG among the groups to ensure the differences described during anesthesia emergence were not due to predisposed differences. This helps us attribute our findings during anesthesia emergence to the substance and to heterogeneous samples. The ROR, in contrast to LOR, is an internal and slow process, primarily determined by pharmacokinetic properties of the maintenance anesthetics. Drug redistribution and elimination from tissues dependent on the cumulatively administered amount (propofol), or alveolar ventilation (volatiles) $[34,35]$. The state change during ROR back to consciousness may include the task of overcoming the orexinergic blockade and hence emergence may even be deemed an active process [36]. Hence, the substance-specific differences in the EEG towards ROR may be caused by the different modes of action $[37,38]$ and the basic pharmacokinetic properties 
of the administered anesthetics [39]. Although both anesthetics seem to modulated the activity in the thalamocortical loop [37], the impact of propofol and sevoflurane on these structures may be different [38] as it may be for isoflurane as well [40]. The EEG features between these anesthetics are different as well under general anesthesia without $[10,11]$ and with burst suppression [20,41]. The effect of propofol on the cortex may be less pronounced [38,42]. If volatiles affect the cortical activity in a stronger fashion, the changes during emergence may be stronger as well, possibly leading to the activation of higher frequencies. The EEG changed differently for the anesthetic regimen investigated with the inhalational anesthetics causing an activation of EEG frequencies in the EEG beta-band range earlier than propofol. At the beginning of the emergence period, the observed strong alpha band activity most probably reflected the described alpha anteriorization during general anesthesia and the hyperpolarized thalamocortical state which also is typical for general anesthesia [43]. The beta activation may point to an activation of cortical activity, but more research needs to be conducted to thoroughly identify possible mechanisms. Further, the "quality" of activation needs to be assessed in the future to be able to distinguish between states of increased activation or higher entropy and states of organized complexity. For instance, low doses of propofol may also lead to increases in (Lempel-Ziv) complexity when compared to an awake baseline [44]. Because the role of neural inertia seems to become more important when investigating state transitions, we need to better understand the mechanisms of the single state transitions [45]. The parameter values were significantly higher after LOR than before ROR. This has been shown for the CSI before [46] at similar time points. This difference may also be attributed to neural inertia.

\subsection{Impact on EEG-Based Monitoring}

Of course, the difference in the emergence EEG characteristics also influence parameters or indices used for monitoring. Here we investigated the impact on PeEn, an entropic measure in the time domain that showed high performance in the separation between consciousness and unconsciousness [26] and the CSI, an index derived from spectral EEG parameters that is still used for patient monitoring. Although, there is no new version of the CSI available, the monitoring performance of this index is comparable to other devices $[47,48]$. For PeEn calculation, the upper limit of the EEG frequency range was $30 \mathrm{~Hz}$ to reduce the influence of possible EMG contamination [49]. We would like to state that monitoring the depth of anesthesia and tracking the state transitions might present different challenges to the monitors. To display the level of anesthesia a smooth and stable index may seem preferable, but it seems to come at the cost of a time delay of index calculation $[9,50]$. Therefore, the monitors may not be designed to reliably track the state transition or identify the time point of LOR or ROR. Still, the evaluation of the emergence behavior may be important in terms of outcome [5] and hence, the index reaction also seems of importance.

We observed similar results with PeEn and with CSI. Still, PeEn showed poorer performance during LOR. This may be due to the reduced frequency range of PeEn and the possible inclusion of EMG activity by the CSI. The CSM EMG index showed a very strong reaction on the LOR (Figure S3). In general, EMG seems to drive neuromonitoring devices to a substantial degree [51] and the EMG frequency range is not limited to frequencies above $30 \mathrm{~Hz}$ but can overlap with the EEG spectrum to a substantial degree [52]. Hence, our analyses may be influenced by motor activity, but the exact impact of EMG needs to be investigated using more sophisticated EMG montages to be able to separate EEG from EMG by e.g., independent component analysis. During ROR, the parameters seemed to perform best for propofol. This was mainly due to the observation that for the inhalational anesthetics, the parameters indicated values reflecting wakefulness prior to ROR, which was most probably caused by the earlier activation of higher frequencies as indicated by the DSA and the CSI $\geq 80$. Consecutively, by choosing the ROR-15s to ROR+30s window, the calculated parameters did not reflect the clinically verified state transition. It has to be stated that our choice of time points before and after the state transition lead to a very challenging data set because the EEG and CSI information were extracted close to the transition. However, at the 
same time this approach possibly most realistically reflects the challenges for the monitors and parameters in daily life. The selection of time points of the EEG or index values to be used for comparison can influence the separation performance as reflected by the AUC. For the CSI, for example, the prediction probability, which is equivalent to the AUC [31], was 0.88 for the comparison of steady-state consciousness versus unconsciousness during LOR [47], whereas it was 0.75 for the separation during state transitions [28]. One issue that has to be considered for our substance-specific investigations is the fact that the indices and entropic parameters do not incorporate the information regarding the substance used. Since propofol and volatile anesthetics lead to different intraoperative EEG patterns [10] the starting conditions at the beginning of anesthesia emergence could have been different. The possibility of intraoperative, substance-specific differences, i.e., if the level of cortical depression is comparable, has to be investigated in the future. Considering basic EEG behavior, and differing performance of CSI and PeEn during anesthesia induction, and emergence under prevalent study conditions, basic pharmacologic aspects have to be considered. Co-administered opioids affect clinical signs of anesthesia emergence [53]. Our study protocol (volatiles/sufentanil vs. propofol/remifentanil) did not eliminate the potentially distorting effect of different opioids on LOR/ROR detection. Thus, it seems appropriate to refer to group-specific instead of substance-specific CSI and PeEn performances. Group-specific differences in the performance to detect ROR may have different reasons. Different substances, i.e., propofol and volatile anesthetics lead to different EEG characteristics during general anesthesia [10]. The spectral EEG characteristics between sevoflurane and isoflurane seem comparable [54]. Further, our result of different durations from CSI indicating awake until the patients' response to the squeeze hand command seems to indicate a substance-specific difference in the time from the evolution of faster EEG oscillations indicative of cortical activity and a coordinated response by the patient. Our results suggest that this episode of uncoordinated cortical activity maybe longer with isoflurane and sevoflurane. A third possibility, which has to be evaluated in the future, may be different emergence trajectories for different substances. Previous research described different spectral EEG trajectories during anesthesia emergence and their resemblance to sleep stages [19] as well as their association with adverse outcomes [18,55]. EEG filter settings of the original study, however, did not allow classifying these trajectories.

\subsection{Clinical Implications}

We could identify anesthesia-regimen-specific differences in the behavior of processed EEG parameters during anesthesia emergence that may be attributed to a difference in the activation of higher EEG frequencies. These differences could lead to the impression of awake patients considerably before the ROR in our patients receiving volatile anesthetics. In case of reacting to this "awake information" and trying to address and wake up the patient, the natural process of emergence may be disrupted. Since the quality of emergence may influence the postoperative cognitive outcome [5], this could be counterproductive. Hence, the anesthesiologist should not only rely on the processed EEG information as a marker for a patient that returns to consciousness.

\subsection{Limitations}

First, our EEG was recorded with the CSM, which has a high pass at $\sim 6 \mathrm{~Hz}$. Hence, we cannot describe any effects in the EEG delta and low-theta band. Especially, the behavior of delta-band activity should be investigated in the future, because it is an important feature of the EEG under general anesthesia [56]. These low frequency ranges are considerably affected by general anesthetics. However, the changes observed in the available frequency range underlines the need for monitoring approaches adjusted to the anesthetic protocol and patient characteristics. Further, we can only report changes in a frontal, single-channel EEG that may limit the amount of information regarding changes in global brain electrical activity. Especially during state transitions, the EEG may be contaminated by muscle activity. Hence, we limited the frequency range for PeEn analysis to $30 \mathrm{~Hz}$. Further, the CSI 
is not as commonly used as for example the BIS or the SEDLine. Nevertheless, it is derived by sub-parameters that process spectral information similar to the other monitors. Our results presented are based on the investigations of different anesthetic regimens that also contain different analgesic drugs, i.e., remifentanil and sufentanil. Hence, the impact of this difference in the regimens has to be investigated in the future. Previous results may suggest that the different opioids may not influence the performance of the bispectral index at the loss of consciousness in a different fashion [56]. Because we present results of the processed EEG, we cannot reveal any mechanistic processes during LOR or ROR. Another factor that needs to be considered in future research is the role of blood pressure on the EEG during anesthesia emergence.

\section{Conclusions}

The EEG features during anesthesia emergence differ, depending on the anesthetic maintenance regimen. The EEG during emergence from propofol anesthesia with remifentanil takes a different course compared to sevoflurane or isoflurane with sufentanil. Patients that received these inhalational anesthetics showed an EEG activation of higher EEG frequencies way before their clinically verified return of responsiveness. These differences influence the EEG based monitoring as well. To more reliably track the patients' emergence in the future, the monitoring approaches may have to be adjusted for the anesthetic regimen used.

Supplementary Materials: The following supporting information can be downloaded at: https: / / www.mdpi.com/article/10.3390/brainsci12010037/s1, Figure S1: AUC matrices derived from the comparisons of the EEG during propofol-induced LOR between the different maintenance groups, Figure S2: Density spectral arrays (relative power) plots for all three groups derived from the pooled (median) relative power spectral density of all patients within a group, Figure S3: Association between index performances based on AUC analysis for the EMG index over the course of 600 s, Figure S4: Difference in CSI.

Author Contributions: Conceptualization, T.K., G.S. and S.P.; methodology, M.K. and S.P.; software, M.K.; data collection, T.K. and S.P.; data analysis, M.K., L.E. and M.L.; discussion of the results: M.K., T.K., M.L., L.E., G.S. and S.P.; writing-original draft preparation, M.K. and S.P.; writing-review and editing, M.K., T.K., M.L., L.E., G.S. and S.P. All authors have read and agreed to the published version of the manuscript.

Funding: The study was funded by departmental sources.

Institutional Review Board Statement: The Ethics Committee of the Technical University of Munich, Munich, Germany (Chairman Prof. A. Schömig) approved the study (Ethical Committee $\mathrm{N}^{\circ} 1239 / 05$ ) and we initiated the study on 2 March 2005.

Informed Consent Statement: Informed consent was obtained from all subjects involved in the study.

Data Availability Statement: Data can be requested from the authors.

Conflicts of Interest: The authors declare no conflict of interest.

\section{References}

1. Rampil, I.J. A Primer for EEG Signal Processing in Anesthesia. Anesthesiology 1998, 89, 980-1002. [CrossRef] [PubMed]

2. Drover, D.; Ortega, H.R. Patient state index. Best Pr. Res. Clin. Anaesthesiol. 2006, 20, 121-128. [CrossRef]

3. Viertio-Oja, H.; Maja, V.; Sarkela, M.; Talja, P.; Tenkanen, N.; Tolvanen-Laakso, H.; Paloheimo, M.; Vakkuri, A.; Yli-Hankala, A.; Merilainen, P. Description of the Entropy algorithm as applied in the Datex-Ohmeda S/5 Entropy Module. Acta Anaesthesiol. Scand. 2004, 48, 154-161. [CrossRef]

4. Jensen, E.W.; Litvan, H.; Revuelta, M.; Rodriguez, B.E.; Caminal, P.; Martinez, P.; Vereecke, H.; Struys, M. Cerebral State Index during Propofol Anesthesia: A Comparison with the Bispectral Index and the A-Line ARX Index. Anesthesiology 2006, 105, 28-36. [CrossRef]

5. Hesse, S.; Kreuzer, M.; Hight, D.; Gaskell, A.; Devari, P.; Singh, D.; Taylor, N.; Whalin, M.; Lee, S.; Sleigh, J. Association of electroencephalogram trajectories during emergence from anaesthesia with delirium in the post-anaesthesia care unit: An early sign of postoperative complications. BJA Br. J. Anaesth. 2019, 122, 622-634. [CrossRef] [PubMed] 
6. $\quad$ Fritz, B.A.; Kalarickal, P.L.; Maybrier, H.R.; Muench, M.R.; Dearth, D.; Chen, Y.; Escallier, K.E.; Ben Abdallah, A.; Lin, N.; Avidan, M.S. Intraoperative Electroencephalogram Suppression Predicts Postoperative Delirium. Anesth. Analg. 2016, 122, 234-242. [CrossRef]

7. Aldecoa, C.; Bettelli, G.; Bilotta, F.; Sanders, R.D.; Audisio, R.; Borozdina, A.; Cherubini, A.; Jones, C.; Kehlet, H.; MacLullich, A. European Society of Anaesthesiology evidence-based and consensus-based guideline on postoperative delirium. Eur. J. Anaesthesiol. 2017, 34, 192-214. [CrossRef] [PubMed]

8. Pilge, S.; Zanner, R.; Schneider, G.; Blum, J.; Kreuzer, M.; Kochs, E. Time Delay of Index Calculation: Analysis of Cerebral State, Bispectral, and Narcotrend Indices. Anesthesiology 2006, 104, 488-494. [CrossRef]

9. Zanner, R.; Pilge, S.; Kochs, E.F.; Kreuzer, M.; Schneider, G. Time delay of electroencephalogram index calculation: Analysis of cerebral state, bispectral, and Narcotrend indices using perioperatively recorded electroencephalographic signals. Br. J. Anaesth. 2009, 103, 394-399. [CrossRef] [PubMed]

10. Akeju, O.; Westover, M.B.; Pavone, K.J.; Sampson, A.L.; Hartnack, K.E.; Brown, E.N.; Purdon, P.L. Effects of sevoflurane and propofol on frontal electroencephalogram power and coherence. Anesthesiology 2014, 121, 990-998. [CrossRef]

11. Stankovski, T.; Petkoski, S.; Raeder, J.; Smith, A.F.; McClintock, P.V.; Stefanovska, A. Alterations in the coupling functions between cortical and cardio-respiratory oscillations due to anaesthesia with propofol and sevoflurane. Philos. Trans. R. Soc. A Math. Phys. Eng. Sci. 2016, 374, 20150186. [CrossRef]

12. Purdon, P.L.; Sampson, A.; Pavone, K.J.; Brown, E.N. Clinical Electroencephalography for Anesthesiologists Part I: Background and Basic Signatures. Anesthesiology 2015, 123, 937-960. [CrossRef]

13. Ni, K.; Cooter, M.; Gupta, D.K.; Thomas, J.; Hopkins, T.J.; Miller, T.E.; James, M.L.; Kertai, M.D.; Berger, M. Paradox of age: Older patients receive higher age-adjusted minimum alveolar concentration fractions of volatile anaesthetics yet display higher bispectral index values. Br. J. Anaesth. 2019, 123, 288-297. [CrossRef]

14. Kreuzer, M.; Stern, M.A.; Hight, D.; Berger, S.; Schneider, G.; Sleigh, J.W.; García, P.S. Spectral and Entropic Features Are Altered by Age in the Electroencephalogram in Patients under Sevoflurane Anesthesia. Anesthesiology 2020, 132, 1003-1016. [CrossRef]

15. Purdon, P.; Pavone, K.; Akeju, O.; Smith, A.; Sampson, A.; Lee, J.; Zhou, D.; Solt, K.; Brown, E. The Ageing Brain: Age-dependent changes in the electroencephalogram during propofol and sevoflurane general anaesthesia. Br. J. Anaesth. 2015, 115, i46-i57. [CrossRef]

16. Schultz, A.; Grouven, U.; Zander, I.; Beger, F.A.; Siedenberg, M.; Schultz, B. Age-related effects in the EEG during propofol anaesthesia. Acta Anaesthesiol. Scand. 2004, 48, 27-34. [CrossRef]

17. Obert, D.P.; Schweizer, C.; Zinn, S.; Kratzer, S.; Hight, D.; Sleigh, J.; Schneider, G.; García, P.S.; Kreuzer, M. The influence of age on EEG-based anaesthesia indices. J. Clin. Anesth. 2021, 73, 110325. [CrossRef]

18. Chander, D.; Garcia, P.S.; MacColl, J.N.; Illing, S.; Sleigh, J.W. Electroencephalographic variation during end maintenance and emergence from surgical anesthesia. PLoS ONE 2014, 9, e106291. [CrossRef]

19. Hight, D.F.; Dadok, V.M.; Szeri, A.J.; García, P.S.; Voss, L.; Sleigh, J.W. Emergence from general anesthesia and the sleep-manifold. Front. Syst. Neurosci. 2014, 8, 146. [CrossRef]

20. Fleischmann, A.; Pilge, S.; Kiel, T.; Kratzer, S.; Schneider, G.; Kreuzer, M. Substance-Specific Differences in Human Electroencephalographic Burst Suppression Patterns. Front. Hum. Neurosci. 2018, 12, 368. [CrossRef]

21. Bandt, C.; Pompe, B. Permutation Entropy: A Natural Complexity Measure for Time Series. Phys. Rev. Lett. $2002,88,174102$. [CrossRef] [PubMed]

22. Tunstall, M.E. Detecting wakefulness during general anaesthesia for caesarean section. Br. Med. J. 1977, 1, 1321. [CrossRef]

23. Schnider, T.W.; Minto, C.F.; Shafer, S.L.; Gambus, P.L.; Andresen, C.; Goodale, D.B.; Youngs, E.J. The Influence of Age on Propofol Pharmacodynamics. Anesthesiology 1999, 90, 1502-1516. [CrossRef]

24. Stockmanns, G.; Ningler, M.; Omerovic, A.; Kochs, E.F.; Schneider, G. NeuMonD: A tool for the development of new indicators of anaesthetic effect. Biomed. Tech./Biomed. Eng. 2007, 52, 96-101. [CrossRef]

25. Berger, S.; Schneider, G.; Kochs, E.F.; Jordan, D. Permutation Entropy: Too Complex a Measure for EEG Time Series? Entropy 2017, 19, 692. [CrossRef]

26. Jordan, D.; Stockmanns, G.; Kochs, E.F.; Pilge, S.; Schneider, G. Electroencephalographic Order Pattern Analysis for the Separation of Consciousness and Unconsciousness: An Analysis of Approximate Entropy, Permutation Entropy, Recurrence Rate, and Phase Coupling of Order Recurrence Plots. Anesthesiology 2008, 109, 1014-1022. [CrossRef]

27. Olofsen, E.; Sleigh, J.W.; Dahan, A. Permutation entropy of the electroencephalogram: A measure of anaesthetic drug effect. Br. J. Anaesth. 2008, 101, 810-821. [CrossRef]

28. Pilge, S.; Blum, J.; Kochs, E.F.; Schoniger, S.A.; Kreuzer, M.; Schneider, G. Does the cerebral state index separate consciousness from unconsciousness? Anesth. Analg. 2011, 113, 1403-1410. [CrossRef]

29. Schneider, G.; Hollweck, R.; Ningler, M.; Stockmanns, G.; Kochs, E.F. Detection of consciousness by electroencephalogram and auditory evoked potentials. Anesthesiology 2005, 103, 934-943. [CrossRef]

30. Hentschke, H.; Stüttgen, M.C. Computation of measures of effect size for neuroscience data sets. Eur. J. Neurosci. 2011, 34, 1887-1894. [CrossRef]

31. Jordan, D.; Steiner, M.; Kochs, E.F.; Schneider, G. A Program for Computing the Prediction Probability and the Related Receiver Operating Characteristic Graph. Anesth. Analg. 2010, 111, 1416-1421. [CrossRef] [PubMed]

32. Cardillo, G. Dunn's Test. 2021. Available online: https:/ / github.com/dnafinder/dunn (accessed on 25 November 2021). 
33. Brown, E.N.; Lydic, R.; Schiff, N.D. General Anesthesia, Sleep, and Coma. N. Engl. J. Med. 2010, 363, 2638-2650. [CrossRef] [PubMed]

34. Yasuda, N.; Lockhart, S.H.; Eger, E.I., II; Weiskopf, R.B.; Liu, J.; Laster, M.; Taheri, S.; Peterson, N.A. Comparison of kinetics of sevoflurane and isoflurane in humans. Anesth. Analg. 1991, 72, 316-324. [CrossRef] [PubMed]

35. Jeong, J.S.; Yoon, S.W.; Choi, S.L.; Choi, S.H.; Lee, B.Y.; Jeong, M.A. Comparison of emergence times with different fresh gas flow rates following desflurane anaesthesia. J. Int. Med. Res. 2014, 42, 1285-1293. [CrossRef] [PubMed]

36. Luppi, A.I.; Spindler, L.R.B.; Menon, D.K.; Stamatakis, E.A. The Inert Brain: Explaining Neural Inertia as Post-anaesthetic Sleep Inertia. Front. Neurosci. 2021, 15, 174. [CrossRef] [PubMed]

37. Alkire, M.T.; Hudetz, A.G.; Tononi, G. Consciousness and Anesthesia. Science 2008, 322, 876-880. [CrossRef]

38. Kratzer, S.; Mattusch, C.; Garcia, P.S.; Schmid, S.; Kochs, E.; Rammes, G.; Schneider, G.; Kreuzer, M.; Haseneder, R. Propofol and sevoflurane differentially modulate cortical depolarization following electric stimulation of the ventrobasal thalamus. Front. Comput. Neurosci. 2017, 11, 109. [CrossRef] [PubMed]

39. Hannivoort, L.N.; Absalom, A.R.; Struys, M. The role of pharmacokinetics and pharmacodynamics in clinical anaesthesia practice. Curr. Opin. Anaesthesiol. 2020, 33, 483-489. [CrossRef]

40. Raz, A.; Grady, S.M.; Krause, B.M.; Uhlrich, D.J.; Manning, K.A.; Banks, M.I. Preferential effect of isoflurane on top-down vs bottom-up pathways in sensory cortex. Front. Syst. Neurosci. 2014, 8, 191. [CrossRef]

41. Kenny, J.D.; Westover, M.B.; Ching, S.; Brown, E.N.; Solt, K. Propofol and sevoflurane induce distinct burst suppression patterns in rats. Front. Syst. Neurosci. 2014, 8, 237. [CrossRef]

42. Boisseau, N.; Madany, M.; Staccini, P.; Armando, G.; Martin, F.; Grimaud, D.; Raucoules-Aime, M. Comparison of the effects of sevoflurane and propofol on cortical somatosensory evoked potentials. Br. J. Anaesth. 2002, 88, 785-789. [CrossRef] [PubMed]

43. Vijayan, S.; Ching, S.; Purdon, P.L.; Brown, E.N.; Kopell, N.J. Thalamocortical mechanisms for the anteriorization of alpha rhythms during propofol-induced unconsciousness. J. Neurosci. 2013, 33, 11070-11075. [CrossRef] [PubMed]

44. Boncompte, G.; Medel, V.; Cortínez, L.I.; Ossandón, T. Brain activity complexity has a non-linear relation to the level of propofol sedation. Br. J. Anaesth. 2021, 127, 254-263. [CrossRef] [PubMed]

45. Sepúlveda, P.O.; Tapia, L.F.; Monsalves, S. Neural inertia and differences between loss of and recovery from consciousness during total intravenous anaesthesia: A narrative review. Anaesthesia 2019, 74, 801-809. [CrossRef]

46. Pilge, S.; Blum, J.; Kreuzer, M.; Schneider, G.; Kochs, E. Does the Cerebral State Index differentiate between consciousness and unconsciousness. A prospective patient study. Eur. J. Anaesthesiol. 2006, 23, 28. [CrossRef]

47. Revuelta, M.; Paniagua, P.; Campos, J.M.; Fernandez, J.A.; Martinez, A.; Jospin, M.; Litvan, H. Validation of the index of consciousness during sevoflurane and remifentanil anaesthesia: A comparison with the bispectral index and the cerebral state index. Br. J. Anaesth. 2008, 101, 653-658. [CrossRef] [PubMed]

48. Pilge, S.; Kreuzer, M.; Kochs, E.F.; Zanner, R.; Paprotny, S.; Schneider, G. Monitors of the hypnotic component of anesthesiaCorrelation between bispectral index and cerebral state index. Minerva Anestesiol. 2012, 78, 636-645. [PubMed]

49. Bonhomme, V.; Hans, P. Muscle relaxation and depth of anaesthesia: Where is the missing link? Br. J. Anaesth. 2007, 99, 456-460. [CrossRef]

50. Kreuzer, M.; Zanner, R.; Pilge, S.; Paprotny, S.; Kochs, E.F.; Schneider, G. Time delay of monitors of the hypnotic component of anesthesia: Analysis of state entropy and index of consciousness. Anesth. Analg. 2012, 115, 315-319. [CrossRef]

51. Hayashi, K.; Sawa, T. The fundamental contribution of the electromyogram to a high bispectral index: A postoperative observational study. J. Clin. Monit. Comput. 2019, 33, 1097-1103. [CrossRef]

52. Kamata, K.; Aho, A.; Hagihira, S.; Yli-Hankala, A.; Jäntti, V. Frequency band of EMG in anaesthesia monitoring. Br. J. Anaesth. 2011, 107, 822-823. [CrossRef] [PubMed]

53. Choi, S.H.; Min, K.T.; Lee, J.R.; Choi, K.W.; Han, K.H.; Kim, E.H.; Oh, H.J.; Lee, J.H. Determination of EC95 of remifentanil for smooth emergence from propofol anesthesia in patients undergoing transsphenoidal surgery. J. Neurosurg. Anesthesiol. 2015, 27, 160-166. [CrossRef] [PubMed]

54. Schwender, D.; Daunderer, M.; Klasing, S.; Finsterer, U.; Peter, K. Power spectral analysis of the electroencephalogram during increasing end-expiratory concentrations of isoflurane, desflurane and sevoflurane. Anaesthesia 1998, 53, 335-342. [CrossRef] [PubMed]

55. Mhuircheartaigh, R.N.; Warnaby, C.; Rogers, R.; Jbabdi, S.; Tracey, I. Slow-wave activity saturation and thalamocortical isolation during propofol anesthesia in humans. Sci. Transl. Med. 2013, 5, ra148-ra208. [CrossRef] [PubMed]

56. Lysakowski, C.; Dumont, L.; Pellégrini, M.; Clergue, F.; Tassonyi, E. Effects of fentanyl, alfentanil, remifentanil and sufentanil on loss of consciousness and bispectral index during propofol induction of anaesthesia. BJA Br. J. Anaesth. 2001, 86, 523-527. [CrossRef] 